Z. klin. Chem. u. klin. Biochem.

8. Jg., S. 284-287, Mai 1970

\title{
Über die $\alpha$-Amylase-Ausscheidung im Urin bei gesunden Personen
}

\author{
Von D. Eberhagen, A. İssmer und B. Brandmater \\ Aus der Poliklinik der Universität München \\ (Direktor: Prof. Dr. W. Seitr)
}

(Eingegangen am 11. Dezember 1969)

Die renale Ausscheidungsrate der $\alpha$-Amylase wurde nach zweistündiger Urinsammelperiode bei pankreas- und nierengesunden Personen bestimmt und mit der Amylase-Aktivität des 24-Stdn.-Urins verglichen. $\mathrm{Da}$ beide Bestimmungen zu identischen Werten führten, wird für die praktische Pankreasdiagnostik die wohl in allen Fällen realisierbare Ermittlung der 1-Stdn.-Amylase-Ausscheidung nach zweistündiger Urinsammlung empfohlen. Eine Alters- oder Geschlechtsabhängigkeit der Exkretionsrate ließ sich ebensowenig nachweisen wie eine Beeinflussung durch die Diurese. Die ermittelten Werte gruppieren sich in Form einer Normalverteilüng.

\section{The urinary excretion of $\alpha$-amylase in bealtby persons}

The rate of renal excretion of $\alpha$-amylase in persons with healthy pancreas and kidneys was determined for each two-hour urine sample and compared with the amylase activity of the $24 \mathrm{hr}$. urine. Since both determinations gave identical values, the one hour amylase excretion determined from a two-hour urine sample, which is practicable in all cases, is recommended as the criterion for practical pancreas diagnosis. Thè excretion rate was independent of sex or diuresis. The values recorded show a normal distribution.

Die $\alpha$-Amylase-Aktivität des Harnes steht in Abhängigkeit zu den Schwankungen der Diurese, und ihre diagnostische Beurteilung ist deshalb mit einem erheblichen Unsicherheitsfaktor behaftet, der sich auch durch gleichzeitige Bestimmung des spezifischen Gewichtes als $\mathrm{MaB}$ für die Harnkonzentrierung nur unvollkommen eliminieren läßt. Aus diesem Grunde fordert man (z.B. (1)) zur Abgrenzung einer akuten Pankreatitis die Ermittlung der Amylaseausscheidung im 24-Stdn.-Urin und kann dann auch etwaige tageszeitliche Clearanceschwankungen außer Betracht lassen. Ein derartiges Vorgehen läßt sich jedoch weder im Rahmen eines poliklinischen Ambulatoriums noch in der Notfallsituation des akuten Abdomens realisieren. ZIEvE und Mitarbeiter (2) führten deshalb die Bestimmung der einstündigen Amylaseausscheidung ein, und es wurde in der Folgezeit gezeigt, daß diese Verkürzung der Urin-Sammelperiode keine Beeinträchtigung der Aussagekraft zur Folge hat (3-5). Zwar ergaben sich bei den Untersuchungen von MYRHE und Sirashua (6), Calkins (7) und van RiEt und Hoeke (8) im Laufe eines Tages beträchtliche Änderungen der pro Stunde ausgeschiedenen Enzymmenge, diese konnten jedoch weder mit bestimmten Tageszeiten noch mit den Lebensgewohnheiten der untersuchten Personen (Nahrungsaufnahme, Arbeit, Schlaf usw.) korreliert werden, und die ermittelten Werte blieben stets unterhalb eines bestimmten Grenzwertes.

CalkINS (9) hat in einer weiteren Studie das Verhalten der 1-Stdn.-Amylaseausscheidung auch bei Patienten mit akuter Pankreatitis verfolgt und die relative Treffsicherheit des Testes bestätigt. Er kommt zu der Schlußfolgerung, daß in kürzeren Sammelperioden wiederholte Urinamylasebestimmungen bessere Informationen zur Erkennung und Verlaufskontrolle einer Pankreas- affektion liefern als die Ermittlung der 24:Stdn.-Ausscheidung. Wegen der allgemeinen Bedeutung dieser Frage und der speziellen poliklinischen Situation erschien deshalb eine Úberprüfung der 1-Stdn.-Amylaseausscheidung an der mitteleuropäischen Bevölkerung mit einer hier eingeführten Bestimmungsmethode wünschenswert. Besonders interessierte dabei die Festlegung des Normalbereiches und die Erfassung tageszeitlicher Schwankungen.

\section{Methodik}

Die Bestimmung der $\alpha$-Amylase-Aktivität erfolgte mit der von RICHTERICH und COLOMBo (10) angegebenen amyloklastischen Arbeitsweise, die eine Modifikation der Technik von SMrTH und ROE (11) darstellt. Die Urine kamen unmittelbar nach Beendigung der Sammelperiode zur Verarbeitung. Die Analysenresultate wurden in internationalen Einheiten ausgedrückt ( $\mu \mathrm{Mol}$ Substratumsatz $\cdot$ Min. $^{-1}, 37^{\circ}$ ).

Die untersuchten Alterskollektive wurden aus Studenten, Bundeswehrangehörigen, Krankenhausangestellten und ambulanten $\mathrm{Pa}$ tienten ausgewählt, bei denen keine Anhaltspunkte für Störungen der Verdauungsorgane und der Nierentätigkeit vorlagen. Allen 292 Personen unterstellten wir deshalb eine normale Pankreasfunktion. Bei 85 von ihnen wurde zuerst ein 24-Stdn.-Sammelurin und anschließend ein 2-Stdn.-Nüchternurin gewonnen; in den anderen Fällen waren die Spender der 2-Stdn.- und der 24-Stdn.Urine nicht identisch.

\section{Ergebnisse}

In der Tabelle 1 sind die ermittelten Werte der Amylaseausscheidung im Urin, getrennt nach Geschlecht und Lebensdezennien der Probanden, zusammengestellt. Zwischen keinen der erfaßten Kollektive ergaben sich aufgrund der mittels des t-Testes nach STudenr durchgeführten Signifikanzberechnung Unterschiede, die für eine Alters- oder Geschlechtsabhängigkeit der Ausschei- 
Tab. 1

a-Amylaseausscheidung im Urin von Normalpersonen. Die Angaben geben den Mittelwert der Kollektive mit der einfachen Standardabweichung wieder und sind auf die stündliche Ausscheidungsrate (E/Std.) umgerechnet

\begin{tabular}{|c|c|c|c|c|}
\hline \multirow{3}{*}{$\begin{array}{c}\text { Alter } \\
\text { (Jahre) }\end{array}$} & \multicolumn{4}{|c|}{ a-Amylaseausscheidung im Urin (E/Std.) } \\
\hline & \multicolumn{2}{|c|}{ Frauen } & \multicolumn{2}{|c|}{ Männer } \\
\hline & 2-Stdn.-Urin & 24-Stdn.-Urin & 2-Stdn.-Urin & 24-Stdn.-Urin \\
\hline $20-30$ & $187 \pm 76(n=15)$ & $230 \pm 82(n=15)$ & $254 \pm 70(n=23)$ & $277 \pm 121(n=21)$ \\
\hline $30-40$ & $222 \pm 51(n=14)$ & $239 \pm 82(n=10)$ & $260 \pm 94(n=20)$ & $288 \pm 130(n=15)$ \\
\hline $40-50$ & $246 \pm 84(n=14)$ & $250 \pm 55(n=10)$ & $242 \pm 85(n=15)$ & $244 \pm 84(n=14)$ \\
\hline $50-60$ & $166 \pm 42(n=14)$ & $197 \pm 84(n=15)$ & $205 \pm 72(n=12)$ & $240 \pm 81(n=14)$ \\
\hline $60-70$ & $127 \pm 36(n=11)$ & $187 \pm 79(n=13)$ & $215 \pm 71(n=14)$ & $285 \pm 98(n=13)$ \\
\hline Bereich & $35-415$ & $30-405$ & $60-450$ & $30-550$ \\
\hline
\end{tabular}

dungstate sprechen ( $p$-Werte zwischen $0,9>p>0,8$ und $0,4>p>0,3)$. Die mittlere Ausscheidungsrate der Kollektive jedoch strebte einem beim weiblichen Geschlecht im fünften, beim männlichen Geschlecht bereits im vierten Lebensjahrzehnt liegenden Maximum $z u$ und nahm dann mit zunehmendem Alter wieder $a b$, wenn man von der 24-Stdn.-Uringruppe bei den Männern absieht. Die Ursache dieses "Trends" dürfte in erster Linie in der altersbedingten Änderung der Pankreasfunktion zu suchen sein.

Keine Unterschiede wurden auch zwischen der Amylaseausscheidung/Stunde während der einander zugeordneten 2-Stdn.- und 24-Stdn.-Urinsammelperiode festgestellt. Um dieses im Zusammenhang mit der vorliegenden Fragestellung besonders interessierende Ergebnis näher zu untersuchen, wurde die stündliche Exkretionsrate im 2-Stdn.- und im 24-Stdn.-Urin des gleichen Spenders korreliert (Abb. 1). Aus diesen Werten konnte

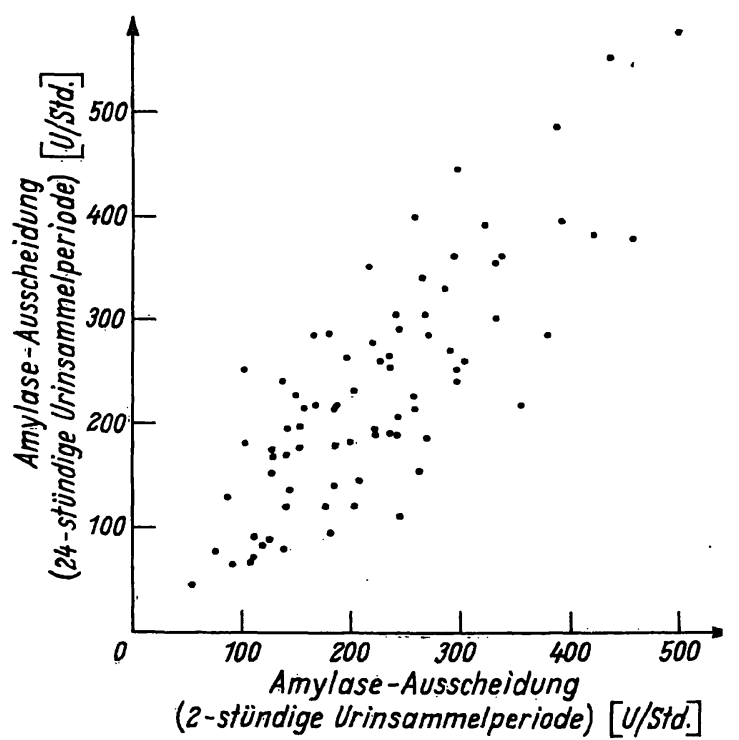

Abb. $1^{\cdot}$

Korrelation der stündlichen Amylase-Exkretionsrate im 2-Stdn.- und im 24-Stdn.-Urin der gleichen Spender

zwar nur ein Korrelationskoeffizient von $r=0,800$ errechnet werden, die Überprüfung der Datenpaare durch den $\mathrm{t}$-Test erbrachte jedoch ein $\mathrm{p}<0,001$.

Zum Ausschluß tagesrhythmischer Schwankungen wurde bei einigen stoffwechselgesunden Personen (Alter zwischen 20 und 30 Jahren) der während eines Tages in 3-Stdn.-Intervallen gesammelte U.rin untersucht und
Tab. 2

Tageszeitliche Schwankungen des Serum-Amylase-Gehaltes und der 1-Stdn.-Ausscheidung der a-Amylase im Harn bei stoffwechselgesunden Personen im Alter zwischen 20 und 30 Jahren

\begin{tabular}{|c|c|c|c|c|c|c|c|}
\hline & & $8^{00}$ & 1100 & 1400 & $17^{00}$ & $20^{00}$ & \\
\hline$\sigma^{\circ}$ & $\begin{array}{l}\text { Urin } \\
\text { Blutserum }\end{array}$ & $\begin{array}{r}201 \\
1104\end{array}$ & $\begin{array}{r}254 \\
1104\end{array}$ & $\begin{array}{r}249 \\
2003\end{array}$ & $\begin{array}{r}290 \\
1086\end{array}$ & $\begin{array}{r}211 \\
1345\end{array}$ & $\begin{array}{l}\text { E/Std. } \\
E / l\end{array}$ \\
\hline q & $\begin{array}{l}\text { Urin } \\
\text { Blutserum }\end{array}$ & $\begin{array}{r}324 \\
2044\end{array}$ & $\begin{array}{r}160 \\
2007\end{array}$ & $\begin{array}{r}153 \\
1929\end{array}$ & $\begin{array}{r}211 \\
1563\end{array}$ & $\begin{array}{r}178 \\
1738\end{array}$ & $\begin{array}{l}\mathrm{E} / \text { Std. } \\
\mathrm{E} / l\end{array}$ \\
\hline$\sigma^{\circ}$ & $\begin{array}{l}\text { Urin } \\
\text { Blutserum }\end{array}$ & $\begin{array}{r}171 \\
1432\end{array}$ & $\begin{array}{r}172 \\
1301\end{array}$ & $\begin{array}{r}212 \\
1706\end{array}$ & $\begin{array}{r}88 \\
1096\end{array}$ & & $\underset{E / l}{\text { E/Std. }}$ \\
\hline 우 & $\begin{array}{l}\text { Urin } \\
\text { Blutserum }\end{array}$ & $\begin{array}{r}124 \\
1437\end{array}$ & $\begin{array}{r}95 \\
1143\end{array}$ & $\begin{array}{r}122 \\
2690\end{array}$ & $\begin{array}{r}149 \\
1678\end{array}$ & & $\begin{array}{l}\text { E/Std. } \\
\mathrm{E} / l\end{array}$ \\
\hline$\sigma^{*}$ & $\begin{array}{l}\text { Urin } \\
\text { Blutserum }\end{array}$ & $\begin{array}{r}269 \\
1163\end{array}$ & $\begin{array}{l}235 \\
857\end{array}$ & $\begin{array}{r}118 \\
1260\end{array}$ & $\begin{array}{r}141 \\
1247\end{array}$ & & $\underset{\mathrm{E} / l}{\mathrm{E} / \mathrm{Std}}$ \\
\hline$\sigma^{\circ}$ & $\begin{array}{l}\text { Urin } \\
\text { Blutserum }\end{array}$ & $\begin{array}{r}262 \\
1898\end{array}$ & $\begin{array}{r}674 \\
2036\end{array}$ & $\begin{array}{r}457 \\
1632\end{array}$ & $\begin{array}{r}376 \\
1675\end{array}$ & & $\underset{\mathrm{E} / l}{\mathrm{E} / \mathrm{S} t \mathrm{l}}$ \\
\hline q & $\begin{array}{l}\text { Urin } \\
\text { Blutserum }\end{array}$ & 1164 & $\begin{array}{r}172 \\
1050\end{array}$ & $\begin{array}{r}194 \\
1003\end{array}$ & $\begin{array}{r}156 \\
1347\end{array}$ & & $\begin{array}{l}\text { E/Std. } \\
\mathrm{E} / l\end{array}$ \\
\hline $0^{\circ}$ & $\begin{array}{l}\text { Urin } \\
\text { Blutserum }\end{array}$ & $\begin{array}{r}275 \\
1422\end{array}$ & $\begin{array}{r}320 \\
1302\end{array}$ & $\begin{array}{r}456 \\
1530\end{array}$ & $\begin{array}{r}403 \\
1437\end{array}$ & & $\underset{\mathrm{E} / l}{\mathrm{E} / S t d .}$ \\
\hline $0^{\circ}$ & $\begin{array}{l}\text { Urin } \\
\text { Blutserum }\end{array}$ & $\begin{array}{r}121 \\
1849\end{array}$ & $\begin{array}{l}163 \\
871\end{array}$ & $\begin{array}{r}419 \\
1946\end{array}$ & $\begin{array}{r}241 \\
1631\end{array}$ & & $\begin{array}{l}\text { E/Std. } \\
\text { E/l }\end{array}$ \\
\hline 0 & $\begin{array}{l}\text { Urin } \\
\text { Blutserum }\end{array}$ & $\begin{array}{r}114 \\
1119\end{array}$ & $\begin{array}{l}276 \\
962\end{array}$ & $\begin{array}{r}161 \\
1025\end{array}$ & $\begin{array}{l}191 \\
999\end{array}$ & & $\underset{E / l}{\text { E/Std. }}$ \\
\hline$\sigma$ & $\begin{array}{l}\text { Urin } \\
\text { Blutserum }\end{array}$ & $\begin{array}{r}306 \\
1479\end{array}$ & $\begin{array}{r}292 \\
1033\end{array}$ & $\begin{array}{r}290 \\
1190\end{array}$ & $\begin{array}{r}365 \\
1001\end{array}$ & & $\underset{E / l}{E / S t d .}$ \\
\hline 우 & $\begin{array}{l}\text { Urin } \\
\text { Blutserum }\end{array}$ & $\begin{array}{r}140 \\
1982\end{array}$ & $\begin{array}{r}253 \\
1969\end{array}$ & $\begin{array}{r}258 \\
1963\end{array}$ & $\begin{array}{r}238 \\
1896\end{array}$ & & $\begin{array}{l}\text { E/Std. } \\
E / l\end{array}$ \\
\hline$\sigma^{\prime}$ & $\begin{array}{l}\text { Urin } \\
\text { Blutserum }\end{array}$ & $\begin{array}{r}147 \\
1337\end{array}$ & $\begin{array}{r}257 \\
1336\end{array}$ & $\begin{array}{r}278 \\
1323\end{array}$ & $\begin{array}{r}478 \\
1480\end{array}$ & & $\underset{E / l}{E / S t d .}$ \\
\hline$\sigma$ & $\begin{array}{l}\text { Urin } \\
\text { Blutserum }\end{array}$ & $\begin{array}{r}190 \\
1507\end{array}$ & $\begin{array}{r}240 \\
1359\end{array}$ & $\begin{array}{r}320 \\
1625\end{array}$ & $\begin{array}{r}308 \\
1783\end{array}$ & & $\begin{array}{l}\text { E/Std. } \\
\mathrm{E} / l\end{array}$ \\
\hline q & $\begin{array}{l}\text { Urin } \\
\text { Blutserum }\end{array}$ & $\begin{array}{r}255 \\
1420\end{array}$ & $\begin{array}{r}261 \\
1451\end{array}$ & $\begin{array}{r}313 \\
1583\end{array}$ & $\begin{array}{r}81 \\
1472\end{array}$ & & $\underset{\mathrm{E} / l}{\text { E/Std. }}$ \\
\hline$\sigma$ & $\begin{array}{l}\text { Urin } \\
\text { Blutserum }\end{array}$ & $\begin{array}{r}290 \\
1385\end{array}$ & $\begin{array}{r}266 \\
1534\end{array}$ & $\begin{array}{r}356 \\
1806\end{array}$ & $\begin{array}{r}352 \\
1780\end{array}$ & & $\begin{array}{l}\text { E/Std. } \\
\mathrm{E} / l\end{array}$ \\
\hline ㅇ & $\begin{array}{l}\text { Urin } \\
\text { Blutserum }\end{array}$ & $\begin{array}{r}305 \\
1704\end{array}$ & $\begin{array}{r}571 \\
1792\end{array}$ & $\begin{array}{r}147 \\
1704\end{array}$ & $\begin{array}{r}171 \\
1669\end{array}$ & & $\underset{E / l}{\text { E/Std. }}$ \\
\hline$\sigma$ & $\begin{array}{l}\text { Urin } \\
\text { Blutserum }\end{array}$ & 1995 & $\begin{array}{r}406 \\
1883\end{array}$ & $\begin{array}{r}419 \\
1845\end{array}$ & $\begin{array}{r}300 \\
1842\end{array}$ & & $\begin{array}{l}\text { E/Std. } \\
E / l\end{array}$ \\
\hline ㅇ & $\begin{array}{l}\text { Urin } \\
\text { Blutserum }\end{array}$ & $\begin{array}{r}102 \\
1446\end{array}$ & $\begin{array}{r}168 \\
1456\end{array}$ & $\begin{array}{r}232 \\
1436\end{array}$ & $\begin{array}{r}141 \\
1331\end{array}$ & & $\begin{array}{l}\text { E/Std. } \\
\text { E/l }\end{array}$ \\
\hline 우 & $\begin{array}{l}\text { Urin } \\
\text { Blutserum }\end{array}$ & 1856 & $\begin{array}{r}185 \\
1770\end{array}$ & $\begin{array}{r}35 \\
2070\end{array}$ & $\begin{array}{r}387 \\
2050\end{array}$ & & $\underset{E / l}{\text { E/Std. }}$ \\
\hline
\end{tabular}

gleichzeitig jeweils eine Blutprobe entnommen. Die ermittelten Werte sind in der Tabelle 2 aufgeführt. Danach wies die $\alpha$-Amylase-Ausscheidungsrate im Urin nicht unerhebliche Schwankungen im Beobachtungszeitraum auf, ohne daß diese jedoch einer bestimmten Tageszeit zugeordnet werden konnten und - abgesehen von einer Ausnahme - den als obere Grenze angesehenen Wert von $450 \mathrm{E} /$ Std. deutlich überschritten. Weiterhin ließen sich keine Beziehungen zur Amylase-Aktivität des Blutserums erkennen. Hier fand sich zwar um $14^{00}$ verschiedentlich ein leicht gegenüber den vorangegangenen und den folgenden Entnahmeterminen erhöhter Aktivitätsgehalt, der postprandial bedingt gewesen sein dürfte, in den meisten Fällen ergab sich jedoch ein ziem- 


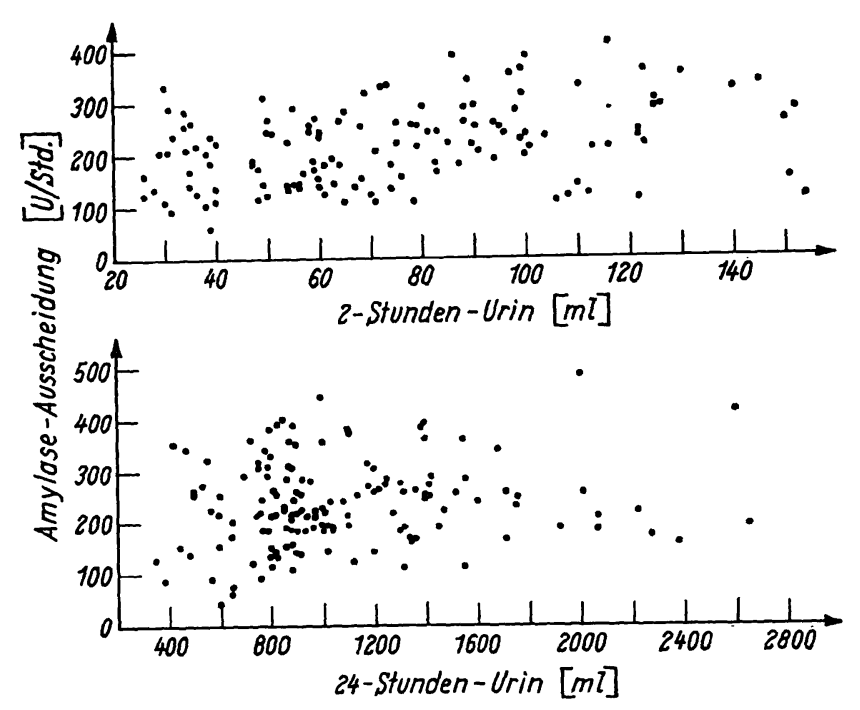

Abb. 2

Darstellung der stündlich ausgeschiedenen $\alpha$-Amylase-Aktivität (E/Std.) in Abhängigkeit von der ausgeschiedenen Urinmenge

lich konstanter Blutspiegel. Nach diesen Ergebnissen brauchen tagesthythmische Schwankungen der AmylaseClearance 'nicht befürchtet $z u$ werden.

Als nächstes sollte der Frage nachgegangen werden, wieweit die 1-Stdn.-Ausscheidung der $\alpha$-Amylase von der Diurese und Antidiurese beeinflußt wird. $\mathrm{Zu}$ diesem Zweck wurde die ermittelte Exkretionsrate gegen das Urinvolumen sowohl bei der 2-Stdn.- als auch bei der 24-Stdn.-Sammelperiode aufgetragen. Wie die Abbildung 2 erkennen läßt, besitzt die Diurese keinen Einfluß auf die Amylaseeliminierungsrate der Nieren. Dieses trifft sowohl für die 24-Stdn.- als auch für die 2-Stdn.-Ausscheidung zu.

Für die statistische Bearbeitung des vorliegenden Materials war die Entscheidung wichtig, nach welcher $\mathrm{Ge}$ setzmäßigkeit sich die gefundenen Werte gruppieren. Zu diesem Zweck wurde der Verteilungstyp sowohl im Histogramm als auch durch Darstellung der prozentualen kumulativen Häufigkeit auf Wahrscheinlichkeitspapier untersucht. Um zu genügend großen Zahlen zu kommen, erfolgte die Trennung der Kollektive nur nach dem Geschlecht. In allen Fällen stellte sich eine eindeutige Normalverteilung dar (Abb. 3), die die Anwendung der entsprechenden statistischen Auswerteregeln rechtfertigte.

\section{Diskussion}

Die unbefriedigende Aussagekraft einer - selbst in Verbindung mit einer Harndichtebestimmung durchgeführten - $\alpha$-Amylase-Bestimmung im Spontanurin hat zu der Auffassung geführt, nur die Amylasewerte des 24-Stdn.-Urins geben verläßliche pankreasdiagnostische Informationen. Ein derartiges Vorgehen läßt sich erklärlicherweise jedoch in vielen Fällen nicht realisieren. Die Untersuchungsergebnisse der vorliegenden Studie zeigen demgegenüber in Übereinstimmung mit den Befunden anderer Autoren (2-5), daß die im 2-Stdn.Urin ermittelte renale Amylase-Exkretionsrate sich nicht von dem im 24-Stdn.-Sammelurin bestimmten Wert unterscheidet, daß beide Werte vielmehr identisch sind. Die obere Grenze der stündlichen Amylase-Ausscheidung liegt bei Anwendung der von RICHTERICH und Colomвo (10) benutzten Technik bei 450 Einheiten, die Minimalausscheidung bei $30 \mathrm{E} / \mathrm{Std}$. Es ließ sich kein Zusammenhang ziwischen dem Urinvolumen und der Amylase-Clearance erkennen; eine Beeinflussung der ermittelten Werte durch die Diurese-Antidiurese braucht demnach nicht befürchtet zu werden. Weiterhin konnte auch eine Alters- und Geschlechtsabhängigkeit ausgeschlossen werden, eine Tatsache, die die Beurteilung des Testergebnisses erleichtert.

Über den Tag verteilte Urin- $\alpha$-Amylase-Bestimmungen ergaben zwar nicht unerhebliche Clearance-Änderungen, tagesrhythmische Schwankungen ließen sich jedoch ausschließen. Die beobachteten Clearance-Änderungen führten nur einmal in den pathologischen Grenzbereich; alle anderen Werte lagen im Normalbereich. Die geringste Streubreite der Einzelwerte fand sich während der morgendlichen Sammelperiode, und man sollte aus diesem Grunde - wenn möglich - dieses Zeitintervall (700 bis $9^{00}$ ) bevorzugen.
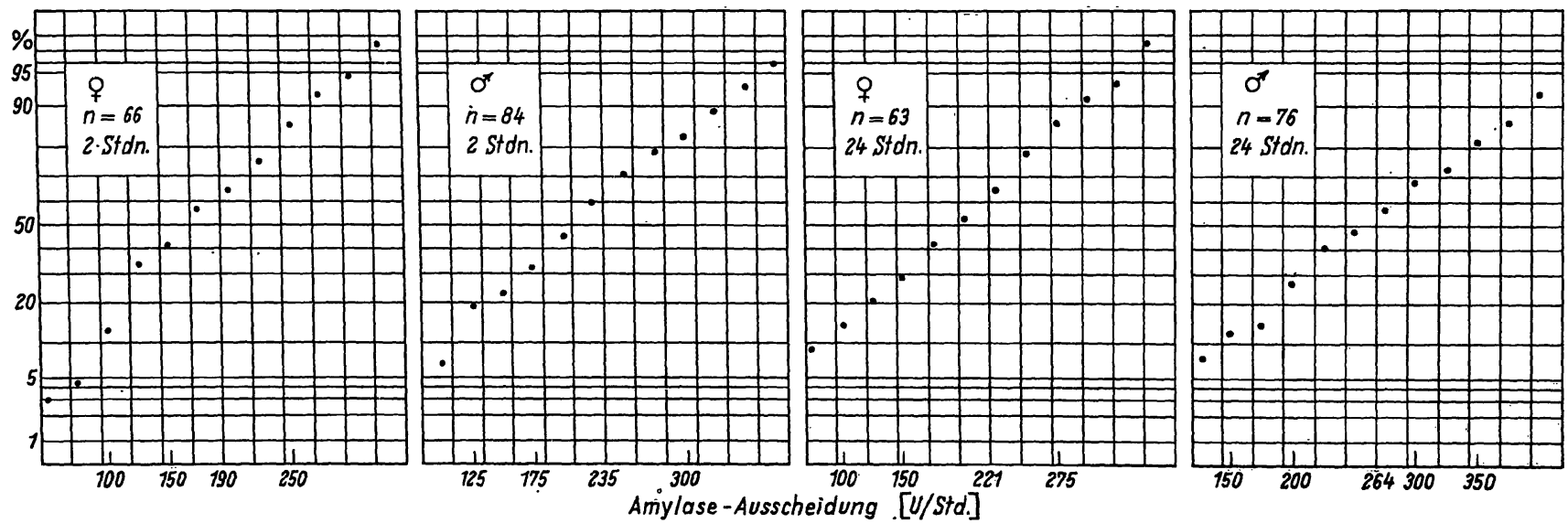

Abb. 3

Darstellung der prozentualen kumulativen Häufigkeit der Amylaseausscheidung auf Wahrscheinlichkeitspapier 
Aus den vorliegenden Ergebnissen möchten wir zwei Konsequenzen ziehen:

1. Urin-Amylase-Bestimmungen sollen heute ausschließlich als Messung der stündlichen renalen Exkretionsrate angelegt werden.
2. Die zweistündige Urin-Sammelperiode ist der vierundzwanzigstündigen hinsichtlich der Aussagekraft des Testes gleichwertig, hinsichtlich der praktischen Durchführbarkeit jedoch eindeutig überlegen und deshalb zu bevorzugen.

\section{Literatur}

1. Osten, W., Dtsch. Med. J. 19, 17 (1968). - 2. Saxon, E. I., W. C. HinkLeY, W. C. Vogel und L. Zieve, Arch. Int. Med., Chicago 99, 607 (1957). - 3. Budd, J. J., Jr., K. E. WALter, M.L. HARRIS und W.A. KNIGHT, Jr., Gastroenterology, Baltimore 36, 333 (1959). - 4. Gambirl, E. E. und H. L. Mason, J. Amer. Med. Ass. 186, 24 (1963). - 5. Gambill, E. E. und H. L. Masom, J. Laborat. Clin. Med., S. Louis 63, 173 (1964). - 6. MYнRE, J. und S. ShashuA, Gastroenterology, Baltimore 48, 836 (1965). 7. Calkins, W. G., Amer. J. Gastro-Enterol., Baltimore 46, 407 (1966). - 8. van Riet, H. G. und J. O. O. Hoeke, Clin. chim. Acta (Amsterdam) 19, 459 (1968). - 9. Calkins, W. G., Amer. J. Gastro-Enterol., Baltimore 49, 415 (1968). - 10. RiCHTERICH, R. und J. P. Colombo, Arztl. Labor 8, 33 (1962). - 11. Smith, B. W. und J. H. ROE, J. biol. Chemistry 227, 357 (1957).

Priv.-Doz. Dr. D. Eberhagen Poliklinik der Universität München Zentrallaboratorium 8000 München 15, Pettenkoferstr. 8a 